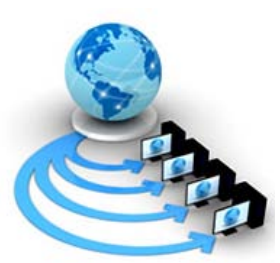

Volume 8, No. 9, November-December 2017

International Journal of Advanced Research in Computer Science

RESEARCH PAPER

Available Online at www.ijarcs.info

\title{
A NETWORK SEGMENT SCHEME FOR BI-DIRECTIONAL DATA BROADCASTING APPROACH IN VANETS
}

\author{
Gourav Gupta \\ Dept. of Computer Science \& Information Technology \\ Sam Higginbottom University of Agriculture,Technology \\ and Sciences (SHUATS) \\ Allahabad ,UP,India
}

\author{
Dr.Tulika \\ Dept. of Computer Science \& Information Technology \\ Sam Higginbottom University of Agriculture,Technology \\ and Sciences (SHUATS) \\ Allahabad ,UP,India
}

\begin{abstract}
In VANETs, the number of vehicles communicates with each other in a network by sending the message from one node to another. While transmission a node can be farther from another node which takes more time with maximum number of hop counts. To enhance the efficiency of the system it is required to reduce the number of hop counts with less delay and more number of packets received at the destination. Considering this fact, a novel approach has proposed in this paper which focused on the essential parameters in the vehicular ad hoc network such as PDR and Delay. In the proposed method, the whole network is divided to reduce the number of delays and high Packet Delivery Ratio at the destination. For the simulation analysis, proposed technique is compared with other traditional FD, EPD, and BDSC protocols which ensure the coherence in the former technique. The results acquired after implementing the techniques are compared in terms of three different parameters i.e. PDR, HOP count and Delay. Moreover, it has concluded that proposed technique offers high packet delivery ratio, less number of hop counts and delay in the network. The PDR of the proposed work is 93.24\% that proffer excel performance in the network.
\end{abstract}

Keywords_-VANETs, Broadcasting, Packet Delivery Ratio, Delay

\section{INTRODUCTION}

For the transmission of data in between the small range of communication in order to obtain the inter-vehicular communication (IVC), the vehicles are required to be installed with the communication devices. Moreover, the surplus of newly developed services that includes vehicle positioned near to each other, like navigation security, games that can be played online, and exchange of information [1]. The scenario of traffic on road is shown through the gateways or internet gateways that can be determined at irregular time periods along with the path that can give large bandwidth connection for a short range.

Due to rapid motion, the cars are in the range of each internet gateway for short duration of time [2]. The internet will not be satisfactory as it only depends on the internet gateways i.e. IGs. The range of vehicles that can communicate with each other can be increased by Ad-hoc networks only. If the VANET network match with the internet connection then the situation raise of Hybrid cooperative networking applications [3]. The optimum VANET network is that which utilize minimum time period for maintaining and controlling overhead. In addition to this, there is a significant issue which is not yet investigated in the fields of VANETs, is shown through the configuration of address. Each vehicle in the VANET must be recognized by distinctive and automatically allocated addresses. The main focus was to hybrid the VANETs with the internet services and the person the vehicle are provided with various kinds of internet services, here the IP address used as natural representative.
All the vehicles in the network must be assigned with distinctive IPv6 address before VANETs linked with internet service. Major benefit associated with VANETs is high transmission rate. Therefore the protocols that come under the IPv6 address configuration, and which are standardized by the IETF are not optimum for VANETs [4]. For instance, in order to achieve the addressing of devices in case of the state full address configuration, the client and server mode can be implemented.

As the vehicle can obtain the address from the distant server, the latency in addressing has been increased. To execute the duplicate address detection i.e. DAD the stateless addressing technique would adapt the discovery procedure used by neighbor configuration standard. As long as duplicate address detection will extend the delay in address configuration then it would be a difficult task to implement to the stateless standards to VANETs network. The IPv6 protocol must be hybrid with the design of geo networking and this has been standardized by European Telecommunication Standards Institute [5]. In order to deploy the addressing the specification defined by ETSI for standardized IPv6 stateless addressing procedure must be utilized. In the standards it is specified that MAC address assigned to vehicles is distinctive. This is the technique which is used to implement the stateless addressing to obtain distinctive IPv6 addresses and duplicate address detection is not used over here. Moreover, it can be assured that the address acquired by the vehicle through stateless addressing technique is distinctive as the MAC address assign to the vehicle cannot be assured to be unique and it is because of following causes: 
- $\quad$ The MAC address can be altered by few commands, for instance: if config, hence it is not certain that the assigned MAC address is unique.

- $\quad$ Assigned media access control address is not mostly globally inimitable.

To distribute the security related information in VANETs the best possible way is to broadcast the message. Flooding of message is an easiest way to distribute the information [6][7]. With the help of Flooding technique the security related messages will be transmitted again by the vehicles and this will be received by all neighboring vehicles. Although, in flooding technique used for broadcasting of messages contains some problems like retransmission of identical message and formation of broadcasting storm [8], the broadcasting storm created due to retransmission of messages the vehicles that have received it and in this way large amount of vehicles starts participating in it. Hence, various researchers had introduced selective retransmission protocol to stop broadcast storm [9]. By implementing the selective retransmission protocol, only few vehicles will transmit again the message and very few vehicles will participate in retransmission in this way it will prevent the network from creating broadcast storm [10].

In broadcasting the message is sent to the destination using the intermediate nodes termed as relay nodes. The total distance between these nodes should be less for the efficient routing. The less will be delay the more accurate and proficient network will be. Thus, this paper has focused on selecting less number of hop counts in the network with less delay and high PDR.

\section{BACKGROUND}

In VANETs, data cannot be transmitted directly from one vehicle to another vehicle. In this case, a relay node has used to transmit the data. Thus, a vehicle has to select a nearer relay node to send data from one to another. A relay node may be used for VANET to gather data from the nearby transmitting nodes and forward it to the required destination node. A relay node may be a static RSU (Road Side Unit) or a mobile node. The packet delivery performance is influenced by the nature of the relay node used in the VANET system [11]. But the problem has arises while selecting the relay node as it can be far from the source vehicle which may arisen the delay in the network and the process will become time consuming. As per the traditional work the parameters used for electing the relay node were PDR, Number of hop counts, end-to-end delay. Hence the proposal given to overcome the various issues of the traditional work by dividing the whole areas of the network into sub portions or sections where each section consist of at least one relay node hence the problem of covering the large distance can be overcome in proposed work. Therefore the data will reach to the destination by covering the less distance and subsequently leads to the less delay and high level of packet delivery ratio.

\section{PROPOSED WORK}

After having a review on the traditional work that was done in the field of VANETs to select the relay nodes, in order to increase the packet delivery ratio and reducing the distance covered by the data, it is observed that the traditional work still poses the backlogs which motivates us to develop a new approach in direction of solution to the problem.

In proposed work, the whole area of the network has divided into sub sections which lead to the reduction in the distance covered by the data to reach the destination.

Along with this the proposed model consists of at least one relay node in each divided section. With this concept the sender nodes need not to search for the nearest relay node in the whole network, in fact it can send the data to the related relay node of the same section. Means the subsection will behave as the cluster of nodes in which the relay node as a cluster head.

Parameters used for the evaluation.

Table 1. List of parameters

\begin{tabular}{|c|c|c|}
\hline S.No & Parameters & Values \\
\hline 1. & Area & $200 * 200$ \\
\hline 2. & Nodes in each cluster & 7 \\
\hline 3. & Packet Size & 1000 \\
\hline
\end{tabular}

\section{A. Splitting of network}

In the existing system, the nodes were deployed in the whole network or one section. The selected source and destination node remains in this large area and takes more time to send packet from one to another. Moreover, the consumption of resources was high as it needs to travel in the whole area [12].

\section{B. Split the network into sixteen sections}

In the proposed type of splitting or sixteen sections splitting the whole area is divided into 16 sub parts. In each part, a relay node is selected. To choose a relay node in each cluster, center node has evaluated and that center node is referred as relay node or head of that particular area. This relay node is responsible for the transmission of data from one to another relay node. With this division, the consumption of energy and resources has reduced and limited to a single area. The total distance travelled between each cluster has also decreased.

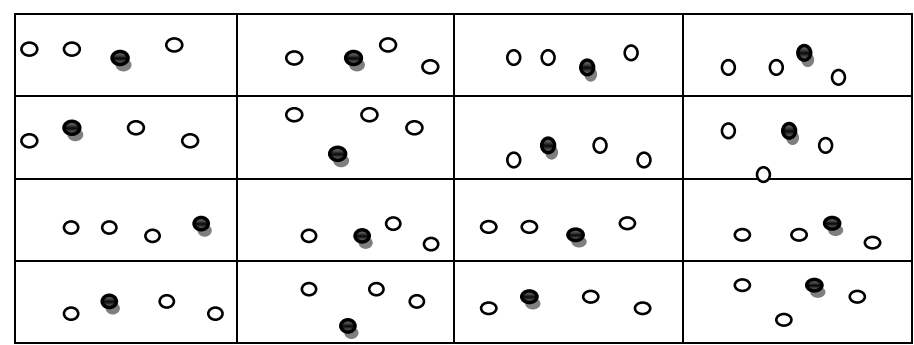

Figure 1 Sixteen Section Splitting 
After initializing the network, spilt the whole network into sub sections. For such purpose, initially evaluate the index1 and index 2 to differ the nodes in each sub section.

Index $1=1$ : nodes: total_nodes;

Index 2 = Nodes: nodes: Total_nodes;

Index 1 and index 2 are basically used to identify which node number resides in which sub section. In order to understand this concept takes an example such as:

$$
\text { Index } 1=1: 5: 20
$$

In the above equation 1 , there are total 20 nodes in the network and 5 depict the total number of nodes in individual section i.e. 5 .

$$
\text { Index } 2=5: 5: 20
$$

The equation 2 depicts another index 2 where 5 to 20 are the total nodes and the step-size 5 is the total nodes in that section. The evaluation of these indexes produces the forthcoming output such as:

$$
\begin{array}{lccc}
\text { Index } 1=1 & 6 & 11 & 16 \\
\text { Index } 2=5 & 10 & 15 & 20
\end{array}
$$

The above acquired output demonstrates that for nodes from 1 to 20, four sections are generated. In each section, total five nodes are resides. The node number 1 to 5 are in the section 1 , 6 to 10 are in section 2, likewise node number 11 to 15 and 16 to 20 are in the section 3 and section 4 respectively. The evaluation of indexes concludes the area in which a particular node number resides.

\section{Relay Node Selection}

After generating subsection of an area, the selection of relay node has done. The relay node in the network will be used to transfer the data from source to the destination. The steps for selecting the relay node is shown in algorithm 3.1

\section{Start}

Evaluate the center node of each area.

Set for loop from 1 to 16

Calculate the Euclidean distance of each node with the attained center node.

$$
\text { Distance }=\sqrt{(\mathrm{x} 1-\mathrm{x} 2)^{2}+(\mathrm{y} 1-\mathrm{y} 2)^{2}}
$$

Save the results into a matrix Arraydist.

Continue for loop for each node End for

Compute minimum distance node with the center. $\min ($ arraydist) ; End

Choose the node with minimum distance as relay node.

After the completion of above step, a relay node finds its corresponding neighbors. For such purpose, it firsts calculates its distance with other relay nodes in other clusters and maintained a table of it. Consequently, all the relay nodes in the network will have their own table in which they contain information about their neighbors.

\section{Route Lookup Phase}

Route lookup is used to find the path from source to destination .Following is used for this purpose.

\section{Start}

Initialize the source in a particular cluster. Set while loop source $\sim=$ Destination Find neighbor of source node.

Select a relay node with minimum distance. Relay node $=$ Minimum (distance);

Next node=relay node; Repeat process until route is not established. End while.

End

\section{RESULTS AND DISCUSSION}

In this section of paper, the results are acquired after performing traditional and the proposed technique and the obtained results are shown below in terms of end-to-end delay, number of hop counts and packet delivery ratio. In the proposed work, the area has divided into subsections where in each sub section there is a relay node which selects the nearer node by looking into its own section rather than the whole network which reduces the overall delay in the network. The comparison analysis has done between different existing techniques such as FD, EPD, BDSC and the proposed technique. All the techniques are compared in terms of different performance parameters such as PDR, Delay and number of hop counts. The results acquired after performing comparison has shown below:

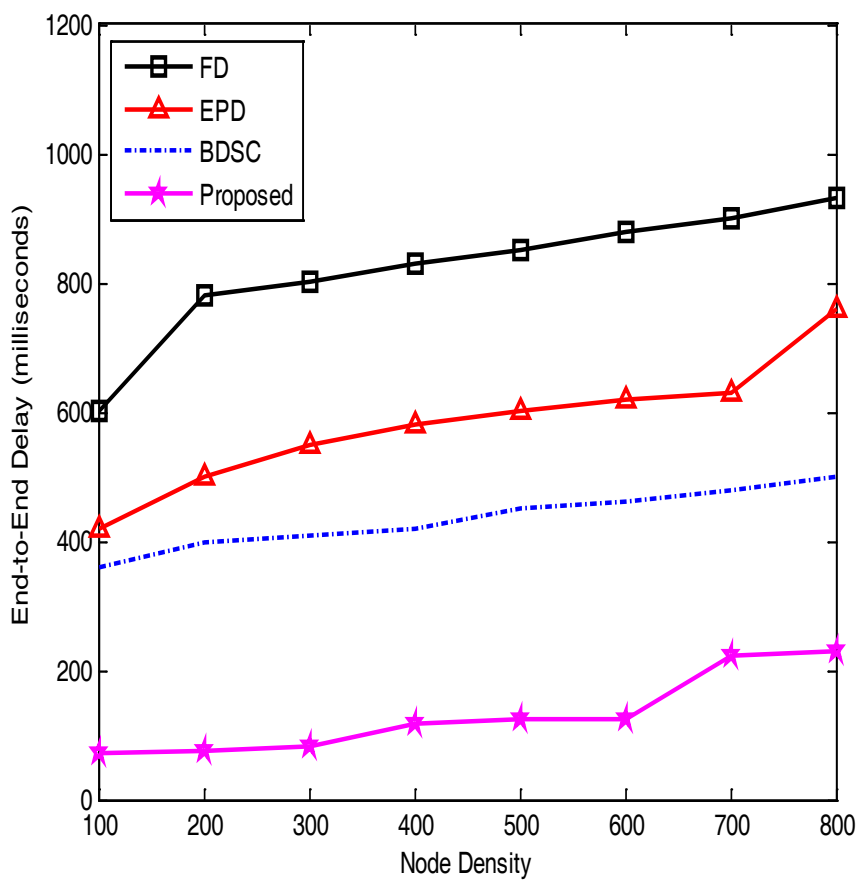

Figure 2 End to End delay of different techniques 
The fig 2 illustrates the end -to-end delay which is calibrated in terms of milliseconds. The graph clearly shows that the proposed technique outperforms in terms of other traditional techniques. The FD has high delay in the network whereas the proposed produces the less delay.

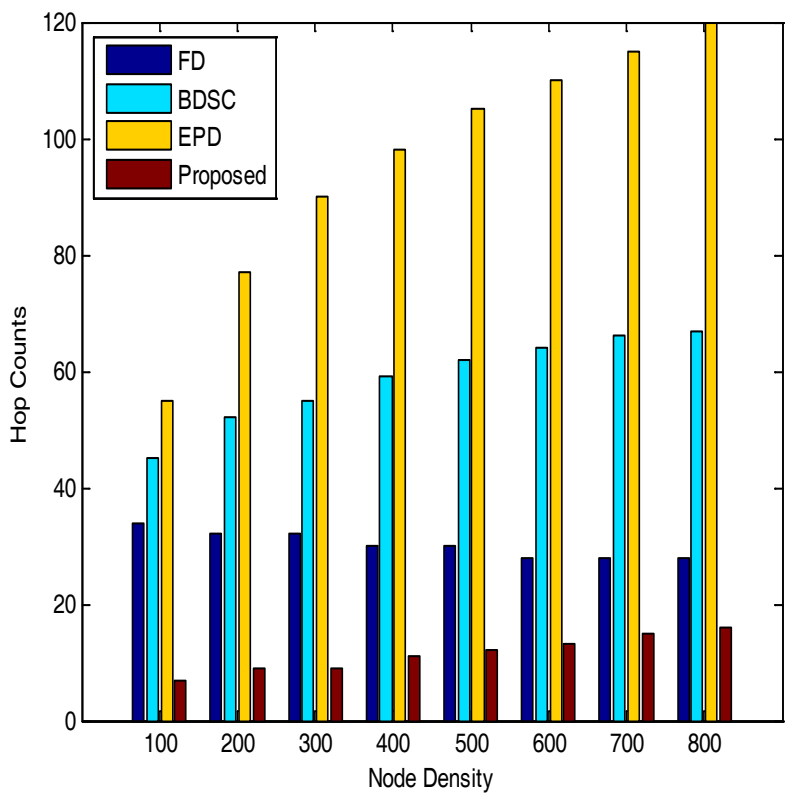

Figure 3 Hop Counts of different techniques

The fig 3 demonstrates the number of hop counts in the network after applying different techniques. From the source to the destination, the relay nodes have chosen and the less number of hop counts in the network the less energy will be consumed by the nodes. Among different techniques, the proposed method outperforms and has less number of relay nodes in defining the path. The highest number of relay nodes in the EPD is the highest and in the proposed method the number of nodes in between the route is lesser.

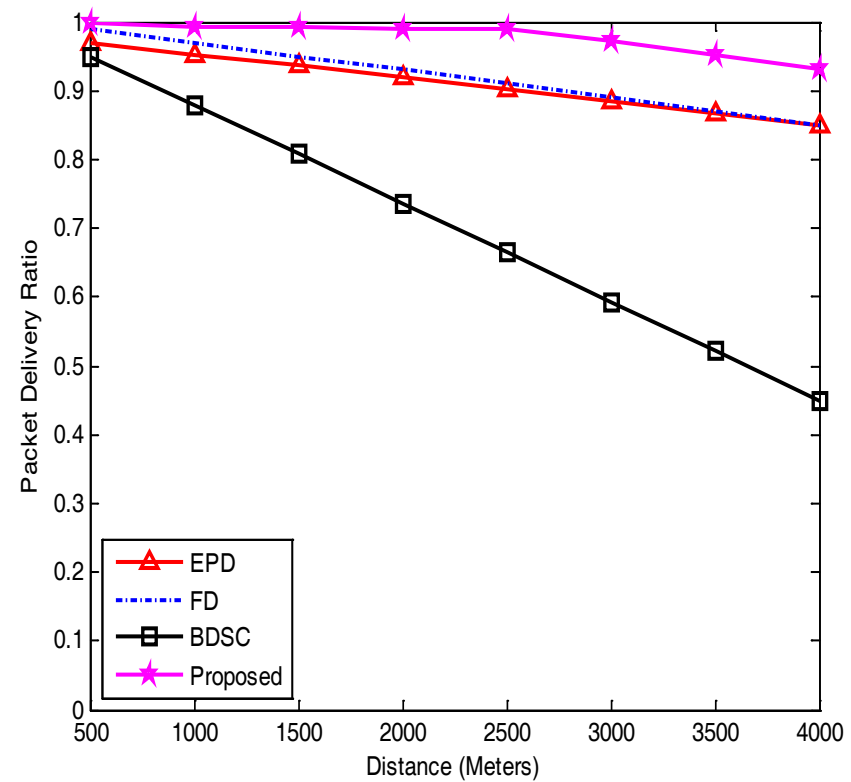

Packet Delivery Ratio of the four different techniques has shown in the fig 4 where using the proposed method, the PDR is high in comparison with other traditional methods. The BDSC has the lowest PDR and with the increase in node density, it has been increasing which is not accurate for the system whereas the proposed method has highest packet delivery ratio which means that at the destination the received number of nodes are quite high.

\section{CONCLUSION AND FUTURE SCOPE}

The main idea behind proposing a new method is to decrease the delay in the network. As in the traditional methods, the delay and the less number of nodes were receiving at the destination so there is a requirement to propose a new method which is capable of reducing the delay and high PDR. In the proposed method, the whole network has divided into small subsections where the nodes sent packet to the nodes which is already in their section rather than the node which is far from it. Considering this idea, the delay has reduced and the number of hop counts in the network. Experimental analysis has performed using the proposed and traditional techniques. Moreover, the comparison has done between traditional FD, EPD, BDSC and the proposed technique. The results acquired from these techniques have shown in terms of different performance parameters such as PDR, End-To-End Delay and number of hop Counts in the network. The simulation analysis ensures that proposed technique outperforms and provides better results such as the number of hop counts in the proposed are higher and lesser in other traditional techniques. Additionally, the delay using proposed method i.e. 123.5935 which is quite less and PDR is 93.13 (\%) i.e. highest among other techniques. In the traditional technique, PDR of BDSC, EPD and FD are 85, 85 and 45 (\%) which is not sufficient for routing in any system.

The proposed method decreases the level of delay in the network by dividing the whole network into subsections. The relay node has selected in the proposed method for the transmission of packet. In future some of the advancements can be done to increase the quality of the system such as: For the selection of relay node in the network, different criteria can be applied so that node with the nearest distance to the sink can be chosen.

The problem of load on nodes can also be considered to avoid the problem of congestion in the network and rely on smoothing transmission of packets between nodes.

\section{REFERENCES}

[1]. X. Wang, D. Le and H. Cheng, "Location-based IPv6 address configuration for vehicular networks", Journal of Network and Systems Management, vol. 24, no. 2, pp. 257-284, 2015.

[2]. Sabih-ur Rehman, "Vehicular ad-Hoc networks (VANETs)—An overview and challenges”, Computer Communications, vol. 48, pp. 141-158, 2014. 
[3]. O.K. Tonguz , N. Wisitpongphan, B. Fan , "DV-CAST: a distributed vehicular broad- cast protocol for vehicular ad hoc networks", Wireless Communication, IEEE, Vol. 17, No. 2, Pp. 47-57, 2010.

[4]. M A. Amoroso , G. Marfia , M. Roccetti , Going realistic and optimal: a distributed multi-hop broadcast algorithm for vehicular safety, Comput. Network, Vol. 55, No.10, pp. 2504-2519, 2011.

[5]. C. Wu , S. Ohzahata , T. Kato , A low latency path diversity mechanism for sender-oriented broadcast protocols in VANETs, Ad Hoc Networks, Vol. 11, No. 7, Pp. 2059-2068, 2013.

[6]. C.-F. Wang , Y.-P. Chiou , G.-H. Liaw , Nexthop selection mechanism for nodes with heterogeneous transmission range in VANETs, Computer Communication, Vol.55, Pp. 22-31, 2015.

[7]. J.A. Sanguesa , M. Fogue , P. Garrido , F.J. Martinez , J.C. Cano , C.T. Calafate , P. Manzoni , RTAD: a real-time adaptive dissemination system for VANETs, Computer Communication, Vol. 60, Pp. 53-70, 2015.
[8]. C. Ming-Chin , C. Meng Chang , DEEP: Density-aware emergency message ex- tension protocol for VANETs, Wireless Communication. IEEE Trans, Vol.12, No. 10, Pp. 4983-4993, 2013.

[9]. X. Wang and H. Qian, "Hierarchical and low-power IPv6 address configuration for wireless sensor networks", International Journal of Communication Systems, vol. 25, no. 12, pp. 1513-1529, 2011.

[10]. X. Wang and S. Zhong, "Research on IPv6 address configuration for a VANET", Journal of Parallel and Distributed Computing, vol. 73, no. 6, pp. 757-766, 2013.

[11]. Osama Rehman, Mohamed Ould-Khaoua and Hadj Bourdoucen, "An adaptive Relay nodes selection scheme for multi-hop broadcast in VANETs", Computer Communications, Pp. 1-15, 2016

[12]. Si-Ho Cha, "A Survey of Broadcast Protocols for Vehicular Adhoc Networks", Smart Computing Review, vol. 4, no. 4, Pp. 246-255, August 2014 